\title{
Article \\ New Aspects for Oscillation of Differential Systems with Mixed Delays and Impulses
}

\author{
Shyam Sundar Santra ${ }^{1,+}+\mathbb{D}$, Khaled Mohamed Khedher ${ }^{2,3,+} \mathbb{D}$ and Shao-Wen Yao ${ }^{4, *,+(\mathbb{D})}$ \\ 1 Department of Mathematics, JIS College of Engineering, Kalyani 741235, India; \\ shyamsundar.santra@jiscollege.ac.in \\ 2 Department of Civil Engineering, College of Engineering, King Khalid University, Abha 61421, Saudi Arabia; \\ kkhedher@kku.edu.sa \\ 3 Department of Civil Engineering, High Institute of Technological Studies, Mrezgua University Campus, \\ Nabeul 8000, Tunisia \\ 4 School of Mathematics and Information Science, Henan Polytechnic University, Jiaozuo 454000, China \\ * Correspondence: yaoshaowen@hpu.edu.cn \\ + These authors contributed equally to this work.
}

check for

updates

Citation: Santra, S.S.; Khedher, K.M.; Yao, S.-W. New Aspects for Oscillation of Differential Systems with Mixed Delays and Impulses. Symmetry 2021, 13, 780. https:// doi.org/10.3390/sym13050780

Academic Editor: Jaume Giné

Received: 12 March 2021

Accepted: 23 April 2021

Published: 1 May 2021

Publisher's Note: MDPI stays neutral with regard to jurisdictional claims in published maps and institutional affiliations.

Copyright: (c) 2021 by the authors. Licensee MDPI, Basel, Switzerland. This article is an open access article distributed under the terms and conditions of the Creative Commons Attribution (CC BY) license (https:// creativecommons.org/licenses/by/ $4.0 /)$.

\begin{abstract}
Oscillation and symmetry play an important role in many applications such as engineering, physics, medicine, and vibration in flight. In this work, we obtain sufficient and necessary conditions for the oscillation of the solutions to a second-order differential equation with impulses and mixed delays when the neutral coefficient lies within $[0,1)$. Furthermore, an examination of the validity of the proposed criteria has been demonstrated via particular examples.
\end{abstract}

Keywords: lebesgue's dominated convergence theorem; neutral; oscillation; nonoscillation; nonlinear

\section{Introduction}

As is well known, impulsive differential equations serve as basic models to study the dynamics of processes that are subject to sudden changes in their states. They appear in the study of many real world problems (see, for instance, [1-3]). We also stress that the modeling of several phenomena is suitably formulated by evolutive partial differential equations and, moreover, moment problem approaches appear as a natural instrument in control theory of neutral type systems; see [4-7], respectively.

Next, we list some recent improvements of oscillation theory for impulsive differential systems.

In [8], the authors considered the impulsive system

$$
\left\{\begin{array}{l}
(z(\eta)-b(\eta) z(\eta-\vartheta))^{\prime}+c(\eta) z\left(\eta-\varsigma_{1}\right)-v(\eta) z\left(\eta-\varsigma_{2}\right)=0, \quad \varsigma_{1} \geq \varsigma_{2}>0 \\
z\left(\varphi_{i}^{+}\right)=I_{i}\left(z\left(\varphi_{i}\right)\right), \quad i \in \mathbb{N}
\end{array}\right.
$$

and obtained several sufficient conditions that ensure the oscillation of the solutions of (1) when $b(\eta) \in P C\left(\left[\eta_{0}, \infty\right), \mathbb{R}_{+}\right)$and $b_{i} \leq \frac{I_{i}(u)}{u} \leq 1$. In [9], the authors considered the problem

$$
\left\{\begin{array}{l}
(z(\eta)-b(\eta) z(\eta-\vartheta))^{\prime}+c(\eta)|z(\eta-\varsigma)|^{\lambda} \operatorname{sgn} z(\eta-\varsigma)=0, \quad \eta \geq \eta_{0} \\
z\left(\varphi_{i}^{+}\right)=b_{i} z\left(\varphi_{i}\right), \quad i \in \mathbb{N}
\end{array}\right.
$$

assuming that $b(\eta) \in P C\left(\left[\eta_{0}, \infty\right), \mathbb{R}_{+}\right)$(that is, $b(\eta)$ is piece-wise continuous in $\left[\eta_{0}, \infty\right)$ ) established sufficient conditions for the oscillation of (2). In [10], Shen and Wang considered the impulsive system

$$
\left\{\begin{array}{l}
z^{\prime}(\eta)+c(\eta) z(\eta-\varsigma)=0, \quad \eta \neq \varphi_{i}, \quad \eta \geq \eta_{0} \\
z\left(\varphi_{i}^{+}\right)-z\left(\varphi_{i}^{-}\right)=I_{i}\left(z\left(\varphi_{i}\right)\right), \quad i \in \mathbb{N}
\end{array}\right.
$$


where $r, I_{i} \in C(\mathbb{R}, \mathbb{R})$ for $i \in \mathbb{N}$ and obtained sufficient conditions for the oscillation of (3).

Oscillatory and non-oscillatory behaviors of a second-order impulsive differential system of neutral type with constant delays and constant coefficients were studied by Tripathy and Santra in [11], where the authors considered the problem

$$
\left\{\begin{array}{l}
(z(\eta)-b z(\eta-\vartheta))^{\prime \prime}+r z(\eta-\varsigma)=0, \quad \eta \neq \varphi_{i}, \quad i \in \mathbb{N} \\
\Delta\left(z\left(\varphi_{i}\right)-b z\left(\varphi_{i}-\vartheta\right)\right)^{\prime}+\tilde{c} z\left(\varphi_{i}-\varsigma\right)=0, \quad i \in \mathbb{N}
\end{array} .\right.
$$

Other sufficient and necessary conditions for the oscillation of second-order impulsive systems of neutral type were found in [12], where Tripathy and Santra studied systems of the form

$$
\left\{\begin{array}{l}
\left(a(\eta)(z(\eta)+b(\eta) z(\eta-\vartheta))^{\prime}\right)^{\prime}+c(\eta) g(z(\eta-\varsigma)), \quad \eta \neq \varphi_{i}, \quad i \in \mathbb{N} \\
\Delta\left(a\left(\varphi_{i}\right)\left(z\left(\varphi_{i}\right)+b\left(\varphi_{i}\right) z\left(\varphi_{i}-\vartheta\right)\right)^{\prime}\right)+c\left(\varphi_{i}\right) g\left(z\left(\varphi_{i}-\varsigma\right)\right)=0, \quad i \in \mathbb{N}
\end{array} .\right.
$$

In [13], the authors found some new sufficient conditions to ensure the oscillation of the impulsive system

$$
\left\{\begin{array}{l}
(z(\eta)-b(\eta) z(\eta-\vartheta))^{\prime}+c(\eta) g(z(\eta-\varsigma))=0, \quad \eta \neq \varphi_{i}, \quad \eta \geq \eta_{0} \\
z\left(\varphi_{i}^{+}\right)=I_{i}\left(z\left(\varphi_{i}\right)\right), \quad i \in \mathbb{N} \\
z\left(\varphi_{i}^{+}-\varsigma\right)=I_{i}\left(z\left(\varphi_{i}-\varsigma\right)\right), \quad i \in \mathbb{N}
\end{array}\right.
$$

for $|b(\eta)|<+\infty$.

In [14], the authors established conditions, both sufficient and necessary, for the oscillation of the following highly nonlinear impulsive differential system of neutral type

$$
\left\{\begin{array}{l}
\left(a(\eta)\left(d^{\prime}(\eta)\right)^{\mu}\right)^{\prime}+\sum_{k=1}^{m} c_{k}(\eta) g_{k}\left(z\left(\varsigma_{k}(\eta)\right)=0, \quad \eta \geq \eta_{0}, \quad \eta \neq \varphi_{i}, \quad i \in \mathbb{N}\right. \\
\Delta\left(a\left(\varphi_{i}\right)\left(d^{\prime}\left(\varphi_{i}\right)\right)^{\mu}\right)+\sum_{k=1}^{m} \widetilde{c}_{k}\left(\varphi_{i}\right) g_{k}\left(z\left(\varsigma_{k}\left(\varphi_{i}\right)\right)\right)=0,
\end{array}\right.
$$

where

$$
d(\eta)=z(\eta)+b(\eta) z(\vartheta(\eta)), \quad \Delta z(a)=\lim _{s \rightarrow a^{+}} z(s)-\lim _{s \rightarrow a^{-}} z(s), \quad-1 \leq b(\eta) \leq 0 .
$$

In [15], the authors obtained oscillation and non-oscillation properties for the solutions to the following class of forced nonlinear neutral impulsive differential systems

$$
\left\{\begin{array}{l}
\left(a(\eta)(z(\eta)+b(\eta) z(\eta-\vartheta))^{\prime}\right)^{\prime}+c(\eta) g(z(\eta-\varsigma))=f(\eta), \quad \eta \neq \varphi_{i}, \quad i \in \mathbb{N} \\
\Delta\left(a\left(\varphi_{i}\right)\left(z\left(\varphi_{i}\right)+b\left(\varphi_{i}\right) z\left(\varphi_{i}-\vartheta\right)\right)^{\prime}\right)+\tilde{c}\left(\varphi_{i}\right) g\left(z\left(\varphi_{i}-\varsigma\right)\right)=\tilde{f}\left(\varphi_{i}\right), \quad i \in \mathbb{N}
\end{array}\right.
$$

for different values of $b(\eta)$ and obtained sufficient conditions for the existence of positive bounded solutions of the above system.

In their recent work [16], Tripathy and Santra studied the following second-order neutral impulsive differential system

$$
\left\{\begin{array}{l}
\left(a(\eta)\left(d^{\prime}(\eta)\right)^{\mu}\right)^{\prime}+\sum_{k=1}^{m} c_{k}(\eta) z^{\mu_{k}}\left(\varsigma_{k}(\eta)\right)=0, \quad \eta \geq \eta_{0}, \quad \eta \neq \varphi_{i} \\
\Delta\left(a\left(\varphi_{i}\right)\left(d^{\prime}\left(\varphi_{i}\right)\right)^{\mu}\right)+\sum_{k=1}^{m} \tilde{c}_{k}\left(\varphi_{i}\right) z^{\mu_{k}}\left(\varsigma_{k}\left(\varphi_{i}\right)\right)=0, \quad i \in \mathbb{N}
\end{array}\right.
$$

where $d(\eta)=z(\eta)+b(\eta) z(\vartheta(\eta))$ and $-1<b(\eta) \leq 0$ and obtained some new oscillation results.

For further details on recent results related to the oscillation theory for ordinary differential equations and for neutral impulsive differential system, we refer the reader to the papers [12,17-33]. In previous studies, most authors studied the oscillation of solutions of the neutral impulsive differential system when the neutral coefficient lies in $(-1,0]$, but 
only a few studied oscillation of solution of the neutral differential system when the neutral coefficient lies in $[0,1)$.

Motivated by the above works, in this study, we aim to establish sufficient and necessary conditions for the oscillation of solutions to the following second-order nonlinear impulsive differential system when the neutral coefficient lies within $[0,1)$ :

$$
\left\{\begin{array}{l}
\left(a(\eta)\left(d^{\prime}(\eta)\right)^{\mu}\right)^{\prime}+c(\eta) z^{v}(\varsigma(\eta))=0, \quad \eta \geq \eta_{0}, \quad \eta \neq \varphi_{i}, i \in \mathbb{N} \\
\Delta\left(a\left(\varphi_{i}\right)\left(d^{\prime}\left(\varphi_{i}\right)\right)^{\mu}\right)+\tilde{c}\left(\varphi_{i}\right) z^{v}\left(\varsigma\left(\varphi_{i}\right)\right)=0
\end{array}\right.
$$

where

$$
d(\eta)=z(\eta)+b(\eta) z(\vartheta(\eta)), \quad \Delta z(g)=\lim _{h \rightarrow g^{+}} z(h)-\lim _{h \rightarrow g^{-}} z(h),
$$

and the functions $b, c, \tilde{c}, \zeta, \vartheta$ are continuous and satisfy the following:

Hypothesis 1 (H1). $\varsigma \in C([0, \infty), \mathbb{R}), \vartheta \in C^{2}([0, \infty), \mathbb{R}), \varsigma(\eta)<\eta, \vartheta(\eta)<\eta, \lim _{\eta \rightarrow \infty} \varsigma(\eta)=$ $\infty, \lim _{\eta \rightarrow \infty} \vartheta(\eta)=\infty$.

Hypothesis $2(\mathbf{H} 2) . \varsigma \in C([0, \infty), \mathbb{R}), \vartheta \in C^{2}([0, \infty), \mathbb{R}), \varsigma(\eta)>\eta, \vartheta(\eta)<\eta, \lim _{\eta \rightarrow \infty} \vartheta(\eta)=$ $\infty$.

Hypothesis 3 (H3). $a \in C^{1}([0, \infty), \mathbb{R})$ with $a(\eta)>0 ; c, \tilde{c} \in C([0, \infty), \mathbb{R})$ with $c(\eta), \tilde{c}(\eta) \geq 0$ for $\eta \geq 0$.

Hypothesis 4 (H4). $b \in C^{2}\left([0, \infty), \mathbb{R}_{+}\right)$with $0 \leq b(\eta) \leq b<1$.

Hypothesis 5 (H5). $\lim _{\eta \rightarrow \infty} A(\eta)=\infty$ where $A(\eta)=\int_{0}^{\eta} a^{-1 / \mu}(s) \mathrm{d}$.

Hypothesis 6 (H6). The sequence $\left\{\varphi_{i}\right\}$ satisfies $0<\varphi_{1}<\varphi_{2}<\cdots \rightarrow \infty$ as $i \rightarrow \infty$; and $\mu$ and $\checkmark$ are the quotient of two odd positive integers.

A solution $z(\eta)$ to (10) is said to be eventually positive (or eventually negative) if there exist $\eta_{1}>0$ such that $z(\eta)>0($ or $z(\eta)<0)$ for $\eta \geq \eta_{1}$.

A differential equation involving an impulse effect is called an impulsive differential equation.

\section{Some Preliminaries}

In this section, we are providing two important lemmas to use in main results.

Lemma 1. Under assumptions (H1)-(H6) for $\eta \geq \eta_{0}$, and $z$ being an eventually positive solution of (10), we have

$$
d(\eta)>0, \quad 0<d^{\prime}(\eta), \quad \text { and } \quad 0 \geq\left(a(\eta)\left(d^{\prime}(\eta)\right)^{\mu}\right)^{\prime} \quad \text { for every } \quad \eta \geq \eta_{1} .
$$

Proof. Let $z$ be an eventually positive solution. Therefore, $d(\eta)>0$ and there exists $\eta_{0} \geq 0$ such that $z(\eta)>0, z(\varsigma(\eta))>0, z(\vartheta(\eta))>0$ for $\eta \geq \eta_{0}$. Then, (10) gives

$$
\begin{gathered}
\left(a(\eta)\left(d^{\prime}(\eta)\right)^{\mu}\right)^{\prime}=-c(\eta) z^{v}(\varsigma(\eta)) \leq 0 \quad \text { for } \eta \neq \varphi_{i}, \\
\Delta\left(a\left(\varphi_{i}\right)\left(d^{\prime}\left(\varphi_{i}\right)\right)^{\mu}\right)=-\tilde{c}\left(\varphi_{i}\right) z^{v}\left(\varsigma\left(\varphi_{i}\right)\right) \leq 0 \quad \text { for } i=1,2, \cdots
\end{gathered}
$$


which shows that $a(\eta)\left(d^{\prime}(\eta)\right)^{\mu}$ is non-increasing for $\eta \geq \eta_{0}$, including jumps of discontinuity. Next, we claim that for $d>0, a(\eta)\left(d^{\prime}(\eta)\right)^{\mu}$ is positive for $\eta \geq \eta_{1}>\eta_{0}$. If not, let $a(\eta)\left(d^{\prime}(\eta)\right)^{\mu} \leq 0$ for $\eta \geq \eta_{1}$; we can choose $c_{1}>0$ such that

$$
a(\eta)\left(d^{\prime}(\eta)\right)^{\mu} \leq-c_{1},
$$

that is,

$$
d^{\prime}(\eta) \leq\left(-c_{1}\right)^{1 / \mu} a^{-1 / \mu}(\eta) .
$$

Integrating both sides from $\eta_{1}$ to $\eta$, we get

$$
d(\eta)-d\left(\eta_{1}\right)-\sum_{i=1}^{\infty} d^{\prime}\left(\varphi_{i}\right) \leq\left(-c_{1}\right)^{1 / \mu}\left(A(\eta)-A\left(\eta_{1}\right)\right) .
$$

Taking the limit of both sides as $\eta \rightarrow \infty$, we have $\lim _{\eta \rightarrow \infty} d(\eta) \leq-\infty$, which leads to a contradiction to $d(\eta)>0$. Hence, $a(\eta)\left(d^{\prime}(\eta)\right)^{\mu}>0$ for $\eta \geq \eta_{1}$, i.e., $d^{\prime}(\eta)>0$ for $\eta \geq \eta_{1}$. Thus, the proof is completed.

Lemma 2. Under assumptions (H1)-(H6) for $\eta \geq \eta_{0}$, and with $z$ being an eventually positive solution of (10), we have

$$
(1-b) d(\eta) \leq z(\eta) \text { for } \eta \geq \eta_{1}
$$

Proof. Let $z$ be an eventually positive solution of (10). Therefore, $d(\eta)>0$ and there exists $\eta \geq \eta_{1}>\eta_{0}$ such that $z(\eta)=d(\eta)-b(\eta) z(\vartheta(\eta)) \geq d(\eta)-b(\eta) d(\vartheta(\eta)) \geq d(\eta)-$ $b(\eta) d(\eta)=(1-b(\eta)) d(\eta) \geq(1-b) d(\eta)$. Hence $d$ satisfies (13) for $\eta \geq \eta_{1}$.

Remark 1. Lemmas 1 and 2 hold for $\mu>v$ and $\mu<v$.

\section{Oscillation Theorems}

In this section, we provide main results to find the sufficient and necessary conditions for the oscillation of solutions to the impulsive system (10).

Theorem 1. Under assumptions (H2)-(H6) for $\eta \geq \eta_{0}$ and $v>\mu$, each solution of (10) is oscillatory if and only if

$$
\int_{0}^{\infty} a^{-1 / \mu}(h)\left[\int_{h}^{\infty} c(g) \mathrm{d} g+\sum_{\varphi_{i} \geq h} \tilde{c}\left(\varphi_{i}\right)\right]^{1 / \mu} \mathrm{d} h=\infty .
$$

Proof. Let $z$ be an eventually positive solution of (10). Therefore, $d(\eta)>0$ and there exists $\eta_{0} \geq 0$ such that $z(\eta)>0, z(\varsigma(\eta))>0, z(\vartheta(\eta))>0$ for $\eta \geq \eta_{0}$. Thus, Lemmas 1 and 2 hold for $\eta \geq \eta_{1}$. Using Lemma 1 and for $\eta_{2}>\eta_{1}$, we have $d^{\prime}(\eta)>0$ for $\eta \geq \eta_{2}$. Thus, for $\eta_{3}>\eta_{2}$ and $c>0$, we have $d(\eta) \geq c$ where $\eta \geq \eta_{3}$. Again, by Lemma 2, we have $z(\eta) \geq(1-b) d(\eta)$ for $\eta \geq \eta_{3}$, and (10) becomes

$$
\begin{gathered}
\left(a(\eta)\left(d^{\prime}(\eta)\right)^{\mu}\right)^{\prime}+c(\eta)((1-b) d(\varsigma(\eta)))^{v} \leq 0 \quad \text { for } \eta \neq \varphi_{i}, \\
\Delta\left(a\left(\varphi_{i}\right)\left(d^{\prime}\left(\varphi_{i}\right)\right)^{\mu}\right)+\tilde{c}\left(\varphi_{i}\right)\left((1-b) d\left(\varsigma\left(\varphi_{i}\right)\right)\right)^{v} \leq 0 \quad \text { for } i=1,2,3, \ldots
\end{gathered}
$$

Integrating (15) from $\eta$ to $+\infty$, we get

$$
\left[a(s)\left(d^{\prime}(h)\right)^{\mu}\right]_{\eta}^{\infty}+\int_{\eta}^{\infty} c(g)((1-b) d(\varsigma(g)))^{v} \mathrm{~d} g+\sum_{\varphi_{i} \geq \eta} \tilde{c}\left(\varphi_{i}\right)\left((1-b) d\left(\varsigma\left(\varphi_{i}\right)\right)\right)^{v} \leq 0 .
$$


Since $a(\eta)\left(d^{\prime}(\eta)\right)^{\mu}$ is positive and non-decreasing. Therefore, $\lim _{\eta \rightarrow \infty} a(\eta)\left(d^{\prime}(\eta)\right)^{\mu}$ finitely exists and is positive.

$$
a(\eta)\left(d^{\prime}(\eta)\right)^{\mu} \geq \int_{\eta}^{\infty} c(g)((1-b) d(\varsigma(g)))^{v} \mathrm{~d} g+\sum_{\varphi_{i} \geq \eta} \tilde{c}\left(\varphi_{i}\right)\left((1-b) d\left(\varsigma\left(\varphi_{i}\right)\right)\right)^{v},
$$

that is,

$$
\begin{aligned}
d^{\prime}(\eta) & \geq a^{-1 / \mu}(\eta)\left[\int_{\eta}^{\infty} c(g)((1-b) d(s(g)))^{v} \mathrm{~d} g+\sum_{\varphi_{i} \geq \eta} \tilde{c}\left(\varphi_{i}\right)\left((1-b) d\left(\varsigma\left(\varphi_{i}\right)\right)\right)^{v}\right]^{1 / \mu} \\
& =(1-b)^{v / \mu} a^{-1 / \mu}(\eta)\left[\int_{\eta}^{\infty} c(g) d^{v}(\varsigma(g)) \mathrm{d} g+\sum_{\varphi_{i} \geq \eta} \tilde{c}\left(\varphi_{i}\right) d^{v}\left(\varsigma\left(\varphi_{i}\right)\right)\right]^{1 / \mu} .
\end{aligned}
$$

Using $(\mathrm{H} 2)$ and with $d(\eta)$ being non-decreasing, we have

$$
d^{\prime}(\eta) \geq(1-b)^{v / \mu} a^{-1 / \mu}(\eta)\left[\int_{\eta}^{\infty} c(g) \mathrm{d} g+\sum_{\varphi_{i} \geq \eta} \tilde{c}\left(\varphi_{i}\right)\right]^{1 / \mu} d^{v / \mu}(\eta),
$$

that is,

$$
\frac{d^{\prime}(\eta)}{d^{v / \mu}(\eta)} \geq(1-b)^{v / \mu} a^{-1 / \mu}(\eta)\left[\int_{\eta}^{\infty} c(g) \mathrm{d} g+\sum_{\varphi_{i} \geq \eta} \tilde{c}\left(\varphi_{i}\right)\right]^{1 / \mu}
$$

Since $v>\mu$. Integrating both sides from $\eta_{3}$ to $+\infty$, we get

$$
(1-b)^{v / \mu} \int_{\eta_{3}}^{\infty} a^{-1 / \mu}(h)\left[\int_{h}^{\infty} c(g) \mathrm{d} g+\sum_{\varphi_{i} \geq \eta} \tilde{c}\left(\varphi_{i}\right)\right]^{1 / \mu} \mathrm{d} h \leq \int_{\eta_{3}}^{\infty} \frac{d^{\prime}(g)}{d^{v / \mu}(g)} \mathrm{d} g<\infty,
$$

which contradicts (14). Thus, the proof of the sufficient part is completed.

Next, we are going to prove the necessary part of the theorem. For this, we assume that (14) does not hold. Hence, for every $\varepsilon>0$ there exists $\eta \geq \eta_{0}$ such that

$$
\int_{\eta}^{\infty} a^{-1 / \mu}(h)\left[\int_{h}^{\infty} c(g) \mathrm{d} g+\sum_{\varphi_{i} \geq h} \tilde{c}\left(\varphi_{i}\right)\right]^{1 / \mu} \mathrm{d} h<\varepsilon \quad \text { for } \quad \eta \geq T
$$

where $2 \varepsilon=\left[\frac{1}{1-b}\right]^{-v / \mu}>0$. We define

$$
S=\left\{z \in C([0, \infty)): \frac{1}{2} \leq z(\eta) \leq \frac{1}{1-b} \text { for } \eta \geq T\right\}
$$

and $\varphi: S \rightarrow S$ as

$$
(\varphi z)(\eta)= \begin{cases}0 & \text { if } \eta \leq T, \\ \frac{1+b}{2(1-b)}-b(\eta) z(\vartheta(\eta)) & \\ +\int_{T}^{\eta} a^{-1 / \mu}(h)\left[\int_{h}^{\infty} c(g) z^{\nu}(\varsigma(g)) \mathrm{d} g+\sum_{\varphi_{i} \geq h} \tilde{c}\left(\varphi_{i}\right) z^{\nu}\left(\varsigma\left(\varphi_{i}\right)\right)\right]^{1 / \mu} \mathrm{d} h & \text { if } \eta>T .\end{cases}
$$

Next, we prove that $(\varphi z)(\eta) \in S$. For $z(\eta) \in S$,

$$
\begin{aligned}
(\varphi z)(\eta) & \leq \frac{1+b}{2(1-b)}+\int_{T}^{\eta} a^{-1 / \mu}(s)\left[\int_{s}^{\infty} c(\psi)\left(\frac{1}{1-b}\right)^{v} \mathrm{~d} \psi+\sum_{\varphi_{i} \geq s} \tilde{c}\left(\varphi_{i}\right)\left(\frac{1}{1-b}\right)^{v}\right]^{1 / \mu} \mathrm{d} s \\
& \leq \frac{1+b}{2(1-b)}+\left(\frac{1}{1-b}\right)^{v / \mu} \cdot \varepsilon=\frac{1+b}{2(1-b)}+\frac{1}{2}=\frac{1}{1-b}
\end{aligned}
$$


and further, for $z(\eta) \in S$

$$
(\varphi z)(\eta) \geq \frac{1+b}{2(1-b)}-b(\eta) \times \frac{1}{1-b}+0 \geq \frac{1+b}{2(1-b)}-\frac{a}{1-b}=\frac{1}{2} .
$$

Hence, $\varphi$ maps from $S$ to $S$.

Now, we define a sequence in $S$ by

$$
\begin{gathered}
u_{0}(\eta)=0 \quad \text { for } \eta \geq \eta_{0}, \\
u_{1}(\eta)=\left(\varphi z_{0}\right)(\eta)= \begin{cases}0 & \text { if } \eta<T \\
\frac{1}{2} & \text { if } \eta \geq T^{\prime}\end{cases} \\
u_{n+1}(\eta)=\left(\varphi z_{n}\right)(\eta) \text { for } n \geq 1, \eta \geq T .
\end{gathered}
$$

Here we see $u_{1}(\eta) \geq u_{0}(\eta)$ for each fixed $\eta$ and $\frac{1}{2} \leq u_{n-1}(\eta) \leq u_{n}(\eta) \leq \frac{1}{1-b}, \quad \eta \geq T$ for $n \geq 1$. Thus, $u_{n}$ converges point-wise to a function $z$. Hence, $z$ is a fixed point of $\varphi$ in $S$ by using Lebesgue's Dominated Convergence Theorem, which proves that there has an eventually positive solution. Thus, the theorem is proved.

Theorem 2. Under assumptions (H1), (H3)-(H6) for $\eta \geq \eta_{0}$ and $v<\mu$, every solution of (10) oscillates if and only if

$$
\left[\int_{0}^{\infty} c(g)[(1-b) A(\varsigma(g))]^{v} \mathrm{~d} g+\sum_{i=1}^{\infty} \tilde{c}\left(\varphi_{i}\right)\left[(1-b) A\left(\varsigma\left(\varphi_{i}\right)\right)\right]^{v}\right]=\infty .
$$

Proof. Let $z(\eta)$ be an eventually positive solution of (10). Then, similar to the proof of Theorem 1, we conclude that (16) holds for $\eta \geq \eta_{2}$, where $\eta_{2}>\eta_{1}>\eta_{0}$. Using (H5), there exists $\eta_{3}>\eta_{2}$ for which $A(\eta)-A\left(\eta_{3}\right) \geq \frac{1}{2} A(\eta)$ for $\eta \geq \eta_{3}$. Integrating (16) from $\eta_{3}$ to $\eta$, we have

$$
\begin{aligned}
d(\eta)-d\left(\eta_{3}\right) & \geq \int_{\eta_{3}}^{\eta} a^{-1 / \mu}(h)\left[\int_{h}^{\infty} c(g)((1-b) d(\varsigma(g)))^{v} \mathrm{~d} g\right. \\
& \left.+\sum_{\varphi_{i} \geq h} \tilde{c}\left(\varphi_{i}\right)\left((1-b) d\left(\varsigma\left(\varphi_{i}\right)\right)\right)^{v}\right]^{1 / \mu} \mathrm{d} h \\
& \geq \int_{\eta_{3}}^{\eta} a^{-1 / \mu}(h)\left[\int_{\eta}^{\infty} c(g)((1-b) d(\varsigma(g)))^{v} \mathrm{~d} g\right. \\
& \left.+\sum_{\varphi_{i} \geq \eta} \tilde{c}\left(\varphi_{i}\right)\left((1-b) d\left(\varsigma\left(\varphi_{i}\right)\right)\right)^{\nu}\right]^{1 / \mu} \mathrm{d} h,
\end{aligned}
$$

that is,

$$
\begin{aligned}
d(\eta) & \geq\left(a(\eta)-a\left(\eta_{3}\right)\right)\left[\int_{\eta}^{\infty} c(g)((1-b) d(\varsigma(g)))^{v} \mathrm{~d} g\right. \\
& \left.+\sum_{\varphi_{i} \geq \eta} \tilde{c}\left(\varphi_{i}\right)\left((1-b) d\left(\varsigma\left(\varphi_{i}\right)\right)\right)^{v}\right]^{1 / \mu} \\
& \geq \frac{1}{2} A(\eta)\left[\int_{\eta}^{\infty} c(g)((1-b) d(\varsigma(g)))^{v} \mathrm{~d} g+\sum_{\varphi_{i} \geq \eta} \tilde{c}\left(\varphi_{i}\right)\left((1-b) d\left(\varsigma\left(\varphi_{i}\right)\right)\right)^{v}\right]^{1 / \mu} .
\end{aligned}
$$

Hence,

$$
d(\eta) \geq \frac{1}{2} A(\eta) \nabla^{1 / \mu}(\eta) \quad \text { for } \quad \eta \geq \eta_{3}
$$


where

$$
\nabla(\eta)=\int_{\eta}^{\infty} c(g)((1-b) d(\varsigma(g)))^{v} \mathrm{~d} g+\sum_{\varphi_{i} \geq \eta} \tilde{c}\left(\varphi_{i}\right)\left((1-b) d\left(\varsigma\left(\varphi_{i}\right)\right)\right)^{v} .
$$

Now,

$$
\begin{aligned}
\nabla^{\prime}(\eta) & =-c(\eta)((1-b) d(\varsigma(\eta)))^{v} \\
& \leq-\frac{1}{2^{v}} c(\eta)[(1-b) A(\varsigma(\eta))]^{v} \nabla^{v / \mu}(\varsigma(\eta)) \leq 0
\end{aligned}
$$

and

$$
\Delta\left(\nabla\left(\varphi_{i}\right)\right)=-\frac{1}{2^{v}} c\left(\varphi_{i}\right)\left[(1-b) A\left(\varsigma\left(\varphi_{i}\right)\right)\right]^{v} \nabla^{v / \mu}\left(\varsigma\left(\varphi_{i}\right)\right) \leq 0 .
$$

From (21), it is clear that $\nabla(\eta)$ is non-increasing in $\left[\eta_{4}, \infty\right)$ and $\lim _{\eta \rightarrow \infty} \nabla(\eta)$ exists. Using (20) and (H1), we find

$$
\begin{aligned}
{\left[\nabla^{1-v / \mu}(\eta)\right]^{\prime} } & =(1-v / \mu) \nabla^{-v / \mu}(\eta) \nabla^{\prime}(\eta) \\
& \leq-\frac{1-v / \mu}{2^{v}} c(\eta)[(1-b) A(\varsigma(\eta))]^{v} \nabla^{v / \mu}(\varsigma(\eta)) \nabla^{-v / \mu}(\eta) \\
& \leq-\frac{1-v / \mu}{2^{v}} c(\eta)[(1-b) A(\varsigma(\eta))]^{v}
\end{aligned}
$$

To estimate the discontinuity of $\nabla^{1-v / \mu}$, we use a Taylor polynomial of order 1 from the function $h(u)=\nabla^{1-v / \mu}$, with $0<v<\mu$, about $u=\tilde{a}$ :

$$
\tilde{b}^{1-v / \mu}-\tilde{a}^{1-v / \mu} \leq(1-v / \mu) \tilde{a}^{-v / \mu}(\tilde{b}-\tilde{a}) .
$$

Then

$$
\Delta\left(\nabla^{1-v / \mu}\left(\varphi_{i}\right)\right) \leq(1-v / \mu) \nabla^{-v / \mu}\left(\varphi_{i}\right) \Delta\left(\nabla\left(\varphi_{i}\right)\right) \leq-\frac{1-v / \mu}{2^{v}} c\left(\varphi_{i}\right)\left[(1-b) A\left(\varsigma\left(\varphi_{i}\right)\right)\right]^{v} .
$$

Integrating (22) from $\eta_{3}$ to $\eta$, we have

$$
\left[\nabla^{1-v / \mu}(h)\right]_{\eta_{4}}^{\eta}-\sum_{\varphi_{i} \geq \eta} \Delta\left[\nabla^{1-v / \mu}\left(\varphi_{i}\right)\right] \leq-\frac{1-v / \mu}{2^{v}} \int_{\eta_{3}}^{\eta} c(h)[(1-b) A(\varsigma(h))]^{v} \mathrm{~d} h,
$$

that is,

$$
\begin{gathered}
\frac{1-v / \mu}{2^{v}}\left[\int_{0}^{\infty} c(h)[(1-b) A(\varsigma(h))]^{v} \mathrm{~d} h+\sum_{i=1}^{\infty} \tilde{c}\left(\varphi_{i}\right)\left[(1-b) A\left(\varsigma\left(\varphi_{i}\right)\right)\right]^{v}\right] \\
\leq-\left[\nabla^{1-v / \mu}(h)\right]_{\eta_{3}}^{\eta}<\nabla^{1-v / \mu}\left(\eta_{3}\right)<\infty
\end{gathered}
$$

which contradicts (17). Thus, the proof is completed.

Example 1. Consider the neutral differential system

$$
\left\{\begin{array}{l}
\left(\left(\left(z(\eta)+e^{-\eta} z(\vartheta(\eta))\right)^{\prime}\right)^{1 / 3}\right)^{\prime}+\eta(z(\eta+2))^{5 / 3}=0 \\
\left(\left(\left(z\left(3^{i}\right)+e^{-3^{i}} z\left(\vartheta\left(3^{i}\right)\right)\right)^{\prime}\right)^{1 / 3}\right)^{\prime}+\left(1+3^{i}\right)\left(z\left(3^{i}+2\right)\right)^{5 / 3}=0 .
\end{array}\right.
$$


Here $v=5 / 3>\mu=1 / 3, a(\eta)=1,0<b(\eta)=e^{-\eta}<1, \varsigma(\eta)=\eta+2, \varphi_{i}=3^{i}$ for $i \in \mathbb{N}$. To check (14), we have

$$
\begin{aligned}
\int_{\eta_{0}}^{\infty}\left[\frac{1}{a(h)}\left[\int_{h}^{\infty} c(g) d g+\sum_{\varphi_{i} \geq h} \tilde{c}\left(\varphi_{i}\right)\right]\right]^{1 / \mu} d h & \geq \int_{\eta_{0}}^{\infty}\left[\frac{1}{a(h)}\left[\int_{h}^{\infty} c(g) d g\right]\right]^{1 / \mu} d h \\
& \geq \int_{2}^{\infty}\left[\int_{h}^{\infty} g d g\right]^{3} d h=\infty .
\end{aligned}
$$

Therefore, each conditions of Theorem 1 hold. Hence, every solution of (23) oscillates.

Example 2. Consider the neutral differential system

$$
\left\{\begin{array}{l}
\left(e^{-\eta}\left(\left(z(\eta)+e^{-\eta} z(\vartheta(\eta))\right)^{\prime}\right)^{3}\right)^{\prime}+\frac{1}{\eta+1}(z(\eta-2))^{7 / 3}=0 \\
\left(e^{-i}\left(\left(z(i)+e^{-i} z(\vartheta(i))\right)^{\prime}\right)^{3}\right)^{\prime}+\frac{1}{i+4}(z(i-2))^{7 / 3}=0
\end{array}\right.
$$

Here, $v=7 / 3<\mu=3, a(\eta)=e^{-\eta}, 0<b(\eta)=e^{-\eta}<1, \varsigma(\eta)=\eta-2, \varphi_{i}=i$ for $i \in \mathbb{N}$, $A(\eta)=\int_{0}^{\eta} e^{s / 3} d s=3\left(e^{\eta / 3}-1\right)$. To check $(17)$, we have

$$
\begin{aligned}
& \frac{1}{2^{v}}\left[\int_{0}^{\infty} c(g)[(1-b) A(\zeta(g))]^{v} d g+\sum_{i=1}^{\infty} \tilde{c}\left(\varphi_{i}\right)\left[(1-b) A\left(\varsigma\left(\varphi_{i}\right)\right)\right]^{v}\right] \\
& \geq \frac{1}{(2)^{7 / 3}} \int_{0}^{\infty} c(g)[(1-b) A(\zeta(g))]^{v} d g \\
& =\frac{1}{(2)^{7 / 3}} \int_{0}^{\infty} \frac{1}{g+1}\left[(1-b) 3\left(e^{(g-2) / 3}-1\right)\right]^{7 / 3} d g=\infty .
\end{aligned}
$$

So, all conditions of Theorem 2 are satisfied. Hence, each solution of (24) oscillates.

\section{Conclusions}

In this work, we tried to establish some new sufficient and necessary conditions for the oscillation of solutions of second-order nonlinear neutral impulsive differential systems with mixed delays of the form (10). Our study is restricted to only when the neutral coefficients $b(\eta)$ lies in $[0,1)$. Still, the problem is open for $-\infty<b(\eta) \leq-1$ and $1 \leq b(\eta)<\infty$. It would be of interest to examine the oscillation of (10) with different neutral coefficients; see, e.g., the papers [34-40] for more details. Furthermore, it is also interesting to analyze the oscillation of (10) with a nonlinear neutral term; see, e.g., the paper [41] for more details.

Author Contributions: Conceptualization, S.S.S., K.M.K. and S.-W.Y.; methodology, S.S.S., K.M.K. and S.-W.Y.; investigation, S.S.S., K.M.K. and S.-W.Y.; resources, S.S.S., K.M.K. and S.-W.Y.; data curation, S.S.S., K.M.K. and S.-W.Y.; writing—original draft preparation, S.S.S., K.M.K. and S.-W.Y.; writing-review and editing, S.S.S., K.M.K. and S.-W.Y.; supervision, S.S.S., K.M.K. and S.-W.Y.; project administration, S.S.S., K.M.K. and S.-W.Y.; funding acquisition, K.M.K. and S.-W.Y. All authors have read and agreed to the published version of the manuscript.

Funding: National Natural Science National Natural Science Foundation of China (No. 71601072), Key Scientific Research Project of Higher Education Institutions in Henan Province of China (No. 20B110006), the Fundamental Research Funds for the Universities of Henan Province (No. NSFRF210314) and Deanship of Scientific Research at King Khalid University for funding this research through the large research groups under grant number RGP. 2/173/42.

Acknowledgments: First of all, we would like to thank reviewers for their careful reading and valuable comments that helped correcting and improving the paper. Below, we listed our responses to the comments and suggestions of the referees. This work is supported by National Natural Science Foundation of China (No. 71601072), Key Scientific Research Project of Higher Education Institutions in Henan Province of China (No. 20B110006) and the Fundamental Research Funds 
for the Universities of Henan Province (No. NSFRF210314). Furthermore, the authors extend their thanks to the Deanship of Scientific Research at King Khalid University for funding this research through the large research groups under grant number RGP. 2/173/42.

Conflicts of Interest: The authors declare no conflict of interest.

\section{References}

1. Bainov, D.D.; Simeonov, P.S. Impulsive Differential Equations: Asymptotic Properties of the Solutions; Series on Advances in Mathematics for Applied Sciences; World Scientific: Singapore, 1995; Volume 28.

2. Lakshmikantham, V.; Bainov, D.D.; Simeonov, P.S. Oscillation Theory of Impulsive Differential Equations; World Scientific: Singapore, 1989 .

3. Agarwal, R.P.; O’Regan, D.; Saker, S.H. Oscillation and Stability of Delay Models in Biology; Springer: New York, NY, USA, 2014.

4. Li, T.; Pintus, N.; Viglialoro, G. Properties of solutions to porous medium problems with different sources and boundary conditions. Z. Angew. Math. Phys. 2019, 70, 86. [CrossRef]

5. Li, T.; Viglialoro, G. Boundedness for a nonlocal reaction chemotaxis model even in the attraction-dominated regime. Differ. Integral Equ. 2011, 34, 315-336.

6. Viglialoro, G.; Woolley, T.E. Solvability of a Keller-Segel system with signal-dependent sensitivity and essentially sublinear production. Appl. Anal. 2020, 99, 2507-2525. [CrossRef]

7. Infusino, M.; Kuhlmann, S. Infinite dimensional moment problem: Open questions and applications. Contemp. Math. 2017, 697, 187-201.

8. Shen, J.; Zou, Z. Oscillation criteria for first-order impulsive differential equations with positive and negative coefficients. J. Comput. Appl. Math. 2008, 217, 28-37. [CrossRef]

9. Graef, J.R.; Shen, J.H.; Stavroulakis, I.P. Oscillation of impulsive neutral delay differential equations. J. Math. Anal. Appl. 2002, 268, 310-333. [CrossRef]

10. Shen, J.H.; Wang, Z.C. Oscillation and asympotic behaviour of solutions of delay differential equations with impulses. Ann. Differ. Eqs. 1994, 10, 61-68.

11. Tripathy, A.K.; Santra, S.S. Characterization of a class of second-order neutral impulsive systems via pulsatile constant. Differ. Equ. Appl. 2017, 9, 87-98. [CrossRef]

12. Tripathy, A.K.; Santra, S.S. Necessary and Sufficient Conditions for Oscillation of a Class of second-order Impulsive Systems. Differ. Equ. Dyn. Syst. 2018. [CrossRef]

13. Santra, S.S.; Tripathy, A.K. On oscillatory first-order nonlinear neutral differential equations with nonlinear impulses. J. Appl. Math. Comput. 2019, 59, 257-270. [CrossRef]

14. Santra, S.S.; Dix, J.G. Necessary and sufficient conditions for the oscillation of solutions to a second-order neutral differential equation with impulses. Nonlinear Stud. 2020, 27, 375-387. [CrossRef]

15. Tripathy, A.K.; Santra, S.S. On the forced impulsive oscillatory nonlinear neutral systems of the second-order. Nonlinear Oscil. 2020, 23, 274-288.

16. Tripathy, A.K.; Santra, S.S. Necessary and sufficient conditions for oscillations to a second-order neutral differential equations with impulses. Kragujev. J. Math. 2023, 47, 81-93.

17. Karpuz, B.; Ocalan, O. Oscillation criteria for a class of first-order forced differential equations under impulse effects. Adv. Dyn. Syst. Appl. 2012, 7, 205-218.

18. Santra, S.S.; Khedher, K.M.; Moaaz, O.; Muhib, A.; Yao, S.-W. Second-order impulsive delay differential systems: Necessary and sufficient conditions for oscillatory or asymptotic behavior. Symmetry 2021, 13, 722. [CrossRef]

19. Santra, S.S.; Ghosh, T.; Bazighifan, O. Explicit Criteria for the Oscillation of Second-Order Differential Equations with Several Sub-linear Neutral Coefficients. Adv. Differ. Equ. 2020, 2020, 643. [CrossRef]

20. Santra, S.S.; Dassios, I.; Ghosh, T. On the asymptotic behavior of a class of second-order non-linear neutral differential Equations with multiple delays. Axioms 2020, 9, 134. [CrossRef]

21. Bazighifan, O.; Ruggieri, M.; Scapellato, A. An Improved Criterion for the Oscillation of Fourth-Order Differential Equations. Mathematics 2020, 8, 610. [CrossRef]

22. Bazighifan, O.; Ruggieri, M.; Santra, S.S.; Scapellato, A. Qualitative Properties of Solutions of Second-Order Neutral Differential Equations. Symmetry 2020, 12, 1520. [CrossRef]

23. Berezansky, L.; Braverman, E. Oscillation of a linear delay impulsive differential equations. Commun. Appl. Nonlinear Anal. 1996, 3, 61-77.

24. Diblik, J.; Svoboda, Z.; Smarda, Z. Retract principle for neutral functional differential equation. Nonlinear Anal. Theory Methods Appl. 2009, 71, 1393-1400. [CrossRef]

25. Diblik, J. Positive solutions of nonlinear delayed differential equations with impulses. Appl. Math. Lett. 2017, 72, 16-22. [CrossRef]

26. Santra, S.S.; Alotaibi, H.; Bazighifan, O. On the qualitative behavior of the solutions to second-order neutral delay differential equations. J. Ineq. Appl. 2020, 2020, 256. [CrossRef]

27. Santra, S.S.; Bazighifan, O.; Ahmad, H.; Chu, Y.-M. Second-Order Differential Equation: Oscillation Theorems and Applications. Math. Probl. Eng. 2020, 2020, 8820066. [CrossRef] 
28. Santra, S.S.; Bazighifan, O.; Ahmad, H.; Yao, S.-W. Second-Order Differential Equation with Multiple Delays: Oscillation Theorems and Applications. Complexity 2020, 2020, 8853745. [CrossRef]

29. Luo, Z.; Jing, Z. Periodic boundary value problem for first-order impulsive functional differential equations. Comput. Math. Appl. 2008, 55, 2094-2107. [CrossRef]

30. Yu, J.; Yan, J. Positive solutions and asymptotic behavior of delay differential equations with nonlinear impulses. J. Math. Anal. Appl. 1997, 207, 388-396.

31. Tripathy, A.K. Oscillation criteria for a class of first-order neutral impulsive differential-difference equations. J. Appl. Anal. Comput. 2014, 4, 89-101.

32. Berezansky, L.; Domoshnitsky, A.; Koplatadze, R. Oscillation, Nonoscillation, Stability and Asymptotic Properties for Second and Higher Order Functional Differential Equations; Chapman \& Hall/CRC Press: Boca Raton, FL, USA, 2020.

33. Santra, S.S.; Majumder, D.; Bhattacharjee, R.; Bazighifan, O.; Khedher, K.; Marin, M. New Theorems for Oscillations to the Differential Equations with Mixed Delays. Symmetry 2021, 13, 367. [CrossRef]

34. Agarwal, R.P.; Bohner, M.; Li, T.; Zhang, C. A new approach in the study of oscillatory behavior of even-order neutral delay differential equations. Appl. Math. Comput. 2013, 225, 787-794. [CrossRef]

35. Bohner, M.; Li, T. Oscillation of second-order $p$-Laplace dynamic equations with a nonpositive neutral coefficient. Appl. Math. Lett. 2014, 37, 72-76. [CrossRef]

36. Chatzarakis, G.E.; Grace, S.R.; Jadlovská, I.; Li, T.; Tunç, E. Oscillation criteria for third-order Emden-Fowler differential equations with unbounded neutral coefficients. Complexity 2019, 2019, 5691758. [CrossRef]

37. Li, T.; Rogovchenko, Y.V. Oscillation of second-order neutral differential equations. Math. Nachr. 2015, 288, 1150-1162. [CrossRef]

38. Li, T.; Rogovchenko, Y.V. Oscillation criteria for even-order neutral differential equations. Appl. Math. Lett. 2016, 61, 35-41. [CrossRef]

39. Li, T.; Rogovchenko, Y.V. Oscillation criteria for second-order superlinear Emden-Fowler neutral differential equations. Monatsh. Math. 2017, 184, 489-500. [CrossRef]

40. Li, T.; Rogovchenko, Y.V. On the asymptotic behavior of solutions to a class of third-order nonlinear neutral differential equations. Appl. Math. Lett. 2020, 105, 106293. [CrossRef]

41. Džurina, J.; Grace, S.R.; Jadlovská, I.; Li, T. Oscillation criteria for second-order Emden-Fowler delay differential equations with a sublinear neutral term. Math. Nachr. 2020, 293, 910-922. [CrossRef] 